\title{
Unselige Union mit der Tabaklobby
}

\author{
Chefredakteur Dr. med. Dirk Einecke
}

\section{„NICHTRAUCHERSCHUTZ“}

\section{Koalition gegen die Gesundheit}

Wahrlich: Die Gesundheit der Bürger ist nicht unbedingt eine Priorität unserer Bundesregierung. Dies zeigt sich einmal mehr an den Fortschritten beim Nichtraucherschutz in öffentlichen Einrichtungen und Gaststätten. Ein völliges Rauchverbot ist seit letzter Woche erst einmal vom Tisch. Vor allem die Union scheint eine schützende Hand über die Tabaklobby zu halten. Nun beraten die Koalitionspartner gar auf der Basis eines Vorschlags der Zigarettenindustrie über „das richtige Maß" beim Nichtraucherschutz, wie die Süddeutsche Zeitung berichtet.

Dabei besteht wissenschaftlich kein Zweifel, wo hier das richtige Maß liegt. Es ist eindeutig, dass Passivrauchen zu erheblichen gesundheitlichen Schäden führt. Gerade erst hat das Deutsche Krebsforschungszentrum (DKFZ) eine Studie zur Schadstoffbelastung in Bars, Diskotheken und Bistros von Fernzügen durchgeführt. Ergebnis: Die ca. 1 Million Arbeitnehmer in der Gastronomie sind als Passivraucher Schadstoffbelastungen ausgesetzt, die in anderen Branchen das Tragen von Atemschutzmasken erfordern würden. Zusam-

\section{AKUTE OTITIS MEDIA}

\section{Abwarten und Antibiotika einsparen}

Eine akute Mittelohrentzündung ist die Diagnose, die bei Kindern am häufigsten eine Antibiotikaverordnung nach sich zieht. In vielen Fällen könnte man den kleinen Patienten jedoch das Antibiotikum ersparen, ohne dadurch das klinische Ergebnis zu verändern. Wie das am besten geht? Man stellt beim Praxisbesuch ein Rezept über ein Antibiotikum aus und fordert die Eltern auf, es erst einzulösen, wenn die Otitis media nach zwei Tagen unverändert fortbesteht oder schlimmer geworden ist. In einer Studie hat man damit gute Erfahrungen gemacht: Von 145 erkrankten Kindern machten $62 \%$ keinen Gebrauch von dem Rezept. Trotzdem bes- men mit allen maßgeblichen wissenschaftlichen Gesellschaften im Herz-Kreislauf-Bereich forderte das DKFZ daher in einem offenen Brief an die Bundeskanzlerin: Öffentliche Gebäude und Gaststätten müssen rauchfrei werden!

Rauchverbot - und schon sinkt die Zahl der Herzinfarkte

Welche frappierenden Sofortauswirkungen ein solches Rauchverbot hat, zeigt eine in „Circulation“ publizierte Studie. In der Arbeiterstadt Pueblo, Colorado/USA, hatten die Behörden vor einiger Zeit ein völliges Rauchverbot in allen öffentlichen Gebäuden sowie am Arbeitsplatz verfügt.

Nun haben Wissenschaftler die Krankenhauseinweisungen wegen akutem Herzinfarkt untersucht. Der Befund ist eindeutig: Im Vergleich zu den 1,5 Jahren vor dem Rauchverbot sank die Infarktrate in den folgenden 1,5 Jahren erheblich und signifikant um $27 \%$. Dies war in einer Nachbarregion ohne Rauchverbot nicht der Fall. Damit bestätigt die Studie eine kleinere Untersuchung aus dem Städtchen Helena in Montana, die vor zwei Jahren publiziert wurde. Kommentar der „American Heart Association“: Der Rückgang der Herzinfarkt-Hospitalisierungsrate ist höchstwahrscheinlich auf die Verminderung des Passivrauchens zurückzuführen.

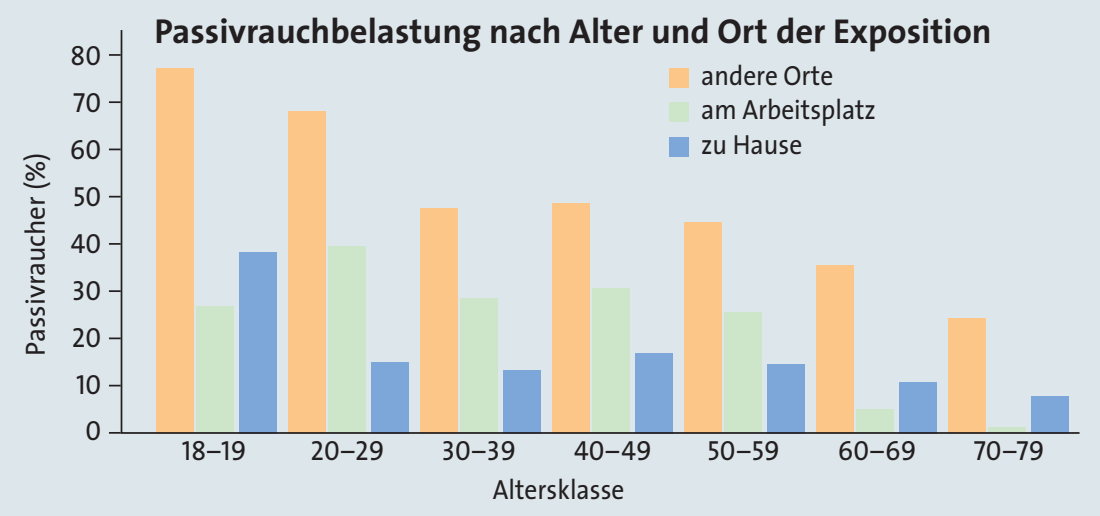

serte sich ihr Gesundheitszustand genauso wie in der Vergleichsgruppe, die sofort ein Antibiotikum erhalten hatte. JAMA 2006;296:1235-41

\section{BODYBUILDING}

\section{Testosteron treibt Hirnzellen in den Selbstmord}

Lästerzungen behaupten, dass bei der Muskelmast der Bodybuilder das Gehirn - so vorhanden - auf der Strecke bleibt. Jetzt gibt es einen wissenschaftlichen Beleg für diese Behauptung. Zumindest in vitro löst ein Überschuss an Testosteron das Extrembodybuilder gerne für Doping zwecke missbrauchen - bei Gehirnzellen die Apoptose aus. Einfach ausgedrückt: Die Gehirnzellen begehen Selbstmord. J Biol Chem 2006;281: 25492-501

\section{ERSTVERSCHREIBUNG EINER THERAPIE}

\section{Sparen Ärzte zu sehr an Erklärungen?}

Um Ihre Patienten vor Einnahmefehlern zu bewahren, sollten Sie ihnen bei der Neuverordnung eines Medikaments fünf Punkte erklären: Name des Medikaments, Zweck der Behandlung, Dauer der Anwendung, mögliche Nebenwirkungen und genaue Dosierungsvorgaben. Dies empfehlen Autoren einer Studie, die Gespräche bei 45 Ärzten aufgezeichnet und dabei festgestellt haben, dass die Informationen bei der Erstverordnung eines Medikaments oft lückenhaft sind. Dauer der Therapie und mögliche Nebenwirkungen wurden sogar nur in einem Drittel der Fälle angesprochen.

Arch Intern Med 2006;166:1855-62 